\title{
Procedural learning across modalities in French-speaking children with specific language impairment
}

\author{
AUDREY GABRIEL and THIERRY MEULEMANS \\ University of Liège \\ CHRISTOPHE PARISSE \\ University of Paris Ouest Nanterre \\ CHRISTELLE MAILLART \\ University of Liège
}

Received: May 4, 2012 Accepted for publication: May 14, 2013

\begin{abstract}
ADDRESS FOR CORRESPONDENCE
Christelle Maillart, Department of Psychology: Cognition and Behavior, University of Liège, B38, Rue de l'Aunaie 30, Liège 4000, Belgium. E-mail: christelle.maillart@ulg.ac.be
\end{abstract}

\begin{abstract}
It has been suggested that the language problems encountered in specific language impairment (SLI) arise from basal ganglia abnormalities that lead to impaired procedural memory. However, recent serial reaction time (SRT) studies did not reveal any differences between the SLI and typically developing (TD) groups on the measures of procedural memory linked to visual sequence learning. In this paper, 16 children with and without SLI were compared on two versions of SRT tasks: a visual task and an auditory one. The results showed that children with SLI were as fast as their TD peers in both modalities. All of the children obtained similar specific sequence learning indices, indicating that they were able to detect regularities in both modalities. Although children with SLI were as accurate as their TD peers for the visual SRT task, they made more errors than their TD peers in auditory SRT conditions. The results indicate that, in relation to procedural memory, the core of the impairment in SLI is not linked to difficulties in the detection of regularities. We argue that when children with SLI present some difficulties, the children's weaknesses might depend on the type of processing involved (e.g., tasks involving auditory sequences).
\end{abstract}

Children with specific language impairment (SLI) encounter difficulties in language acquisition, despite normal intelligence, normal vision and hearing, the absence of neurological impairment, and even growing up in a supportive communicative environment. Several areas of language may be affected, including vocabulary, morphosyntax, discourse, written language, and social language (for reviews, see Leonard, 1998; and Schwartz, 2009). Nevertheless, some researchers question the relevance of the term specific language impairment (Ors, 2002).

(C) Cambridge University Press 2014 0142-7164/14 \$15.00 
Gabriel et al.: Procedural learning in children with SLI

Fernell, Norrelgen, Bozkurt, Hellberg, and Löwing (2002) and Ors (2002) claim that language problems do not occur in isolation. Children with SLI also perform poorly in nonlinguistic domains, such as attention, executive, and motor tasks (Archibald \& Gathercole, 2007; Bishop \& Norbury, 2005; Campbell \& Skarakis-Doyle, 2007; Ellis Weismer, Plante, Jones, \& Tomblin, 2005; Hill, 2001; Hoffman \& Gillam, 2004; Im-Bolter, Johnson, \& Pascual-Leone, 2006; Miller, Kail, Leonard, \& Tomblin, 2001; Noterdaeme, Amorosa, Mildenberger, Sitter, $\&$ Minow, 2001). Therefore, two broad explanatory theories have been proposed to clarify SLI. The first approach focuses on the linguistic aspects of SLI, in particular on specific grammatical structures that might be affected (Ullman \& Gopnik, 1999). The second explanatory approach is based on the view that linguistic impairment in children with SLI may arise from deficient nonlinguistic processing, such as auditory temporal processing (Joanisse \& Seidenberg, 1998, 2003; Tallal, Merzenich, Miller, \& Jenking, 1998), and/or procedural processing (Ullman \& Pierpont, 2005). The current study focuses on both types of processing, especially when they involve sequential aspects.

Children with SLI demonstrate poorer performances than their age-matched peers in various tests of fine and gross motor function (Jancke, Siegenthaler, Preis, \& Steinmetz, 2006; Leonard et al., 2007; Powell \& Bishop, 1992). Their motor deficits appear to be more serious when sequential aspects are involved (Bishop, 2002; Hill, Bishop, \& Nimmo-Smith, 1998). An auditory temporal processing deficit was associated with the language deficits (Leonard, Bortolini, Caselli, McGregor, \& Sabbadini, 1992), especially when perception involved rapid events. The work by Tallal and colleagues (for a review, see Tallal, 2000) showed that children with SLI had difficulties identifying and discriminating successive phonetic elements and nonspeech sound stimuli that were either short in duration or separated by short interstimulus intervals (Tallal \& Piercy, 1974; Tallal \& Stark, 1981; Tallal, Stark, \& Mellits, 1985). However, null findings for SLI groups on such tasks have been reported (Bishop, Adams, Nation, \& Rosen, 2005). Other studies (McArthur \& Bishop, 2004; Mengler, Hogben, Mitchie, \& Bishop, 2005) demonstrated that the poor performance of certain people with SLI on rapid auditory processing tasks may stem from a lesser ability to discriminate between the frequencies of sounds rather than to process rapidly presented sounds.

These results suggest that the sequential information processing deficit may underlie some of the linguistic and nonlinguistic impairments exhibited by children with SLI. The ability to extract rules is already in place at birth (Fromm, Schöler, $\&$ Scherer, 1998). The child does not need to first seek these rules in learning a language but is born with a complete regularities-abstracting system. This ability may be less efficient or impaired in SLI children. The procedural deficit hypothesis (PDH), recently proposed by Ullman and Pierpont (2005), goes one step further and suggests that most of the linguistic (especially grammatical) problems and nonlinguistic deficits observed in SLI children can be understood in terms of an impaired procedural memory system whose functions are compensated by an intact declarative memory system.

Procedural memory can be defined as the memory system in charge of encoding, storing, and retrieving the procedures that underlie motor, verbal, and cognitive skills (Cohen \& Squire, 1980). In training sessions conducted by Doyon et al. 
Gabriel et al.: Procedural learning in children with SLI

(2009), distinct stages were identified during the acquisition of new skills: first, a fast initial learning phase associated with considerable within-session improvement; second, an intermediate consolidation following a latent interval of more than $6 \mathrm{hr}$ after the initial training session; and third, a slow phase in which further gains can be observed across several sessions of practice (Doyon et al., 2009). The PDH proposes that SLI can be largely explained by a dysfunction of fronto/basal ganglia circuits or the cerebellum, whereas the medial temporal lobe structures that underlie learning and consolidation in declarative memory are expected to remain largely unaffected. Procedural memory, which has been discussed in connection with its role in rule construction, implicit learning, sequence processing, and fast automatic recall, is thought to be particularly important for the acquisition and use of skills involving sequences. This system may therefore be involved in the implicit acquisition, storage, and use of knowledge (Gabrieli, 1998; Willingham, 1998) that is sequentially or probabilistically structured (Knowlton, Mangels, \& Squire, 1996). Information learned in this system requires repeated exposure. Unlike learning via the procedural memory system, learning via the declarative memory system can be achieved following a single exposure to the target stimulus. This memory system is considered to be mainly involved in learning, storing, and retrieving general knowledge about the world as well as personal experiences (Eichenbaum, 2000; Squire, Knowlton, \& Musen, 1993), and it may also process the arbitrary binding of conceptual, phonological, and semantic representations.

Not only procedural memory but also nonprocedural functions that depend on the basal ganglia/frontal circuitry, such as auditory processing (especially the processing involving sequential aspects), are predicted to be impaired in the PDH (cf. Ullman \& Pierpont, 2005), leading to deficits in implicit sequential learning and some aspects of grammar. This poor auditory processing is considered to be secondary to basal gangla/frontal anomalies and not directly related to the language problems or procedural memory problems in SLI.

Most studies support the PDH positions across a variety of procedural learning situations, including nonlinguistic (Adi-Japha, Strulovich-Schwartz, \& Julius, 2011; Kemény \& Lukács, 2010; Lum, Conti-Ramsden, Page, \& Ullman, 2012; Lum, Gelgec, \& Conti-Ramsden, 2010; Tomblin, Mainela-Arnold, \& Zhang, 2007) and linguistic tasks (Evans, Saffran, \& Robe-Torres, 2009; Hsu, Christiansen, Tomblin, Zhang, \& Gomez, 2006; Plante, Gomez, \& Gerken, 2002). Usually, implicit visuospatial serial reaction time (SRT) designs are used to explore procedural initial learning abilities. In a typical SRT task, participants are asked to react as quickly and as accurately as possible to stimuli that appear on a computer screen by pressing one of four keys on the keyboard, where each key corresponds to a stimulus location on the screen. Unbeknownst to the participant, the stimulus does not appear randomly but follows a repeated sequence. In such a task, learning of the sequence is shown by longer reaction times (RTs) in a transfer block in which a new sequence of stimuli is presented in contrast with the previous learning block (e.g., Meulemans, Van der Linden, \& Perruchet, 1998).

With the visual paradigm, Tomblin et al. (2007) showed that 15-year-old teenagers with SLI had slower learning rates of the deterministic sequence (i.e., a sequence in which only regularities were inserted) as compared to controls. Group differences in the learning rate on the SRT task were found between high and low 
grammar groups but not between high and low vocabulary groups. Other studies by Lum and colleagues also seem to confirm the predictions of the PDH. Lum et al. (2010) showed that children with SLI did not achieve sequence learning during a deterministic SRT, whereas typically developing (TD) children did. These results were confirmed after removing the variance related to children's motor speed. More recently, Lum et al. (2012) replicated the results obtained in 2010, even when holding working memory constant. Lum et al. (2012) also showed that grammatical abilities were associated with procedural memory in the TD children but with declarative memory in children with SLI. Procedural learning impairment in SLI was reported in the study of procedural graphomotor learning (Adi-Japha, Strulovich-Schwartz, \& Julius, 2011) in a study examining probabilistic category learning (Kemény \& Lukács, 2010) and in the linguistic domain, such as an artificial grammar learning task (Plante et al., 2002) or a statistical learning task (Evans et al., 2009; Hsu et al., 2006).

However, three recent visual SRT studies (Gabriel, Maillart, Guillaume, Stefaniak, \& Meulemans, 2011; Hedenius et al., 2011; Lum \& Bleses, 2012) did not support the expectations of Ullman and Pierpont's (2005) claims, reporting that both the SLI and TD groups demonstrated knowledge of the repeating sequence. In 2011, Gabriel and colleagues showed that children with SLI were able to learn a probabilistic sequence (i.e., a sequence in which some irregularities are inserted; Schvaneveldt \& Gomez, 1998) as fast and as accurately as TD peers and that they presented similar sequence-specific learning indices. Furthermore, the results did not indicate that grammatical abilities were directly related to sequential pattern learning performance. Hedenius et al. (2011) also showed that although children with grammar impairments failed to consolidate sequence learning, they presented intact initial sequential learning during an alternating SRT. A link between grammatical problems in children with SLI and consolidation and long-term sequence retention impairment could not be excluded as suggested by the presence of a positive correlation between grammar knowledge (but not vocabulary knowledge) and sequence knowledge at the follow-up session. The study realized by Lum and Bleses (2012) revealed comparable levels between children with or without SLI on the deterministic procedural memory task used previously (Lum et al., 2010). This study demonstrated that there was no association between impaired grammar abilities and procedural memory.

The predictions of Ullman and Pierpont's model still need to be tested in more detail, including verbal and nonverbal designs. These three recent SRT studies suggest that the core of the impairment of SLI may not be linked to a global dysfunction in procedural learning mechanisms themselves because some visual procedural learning abilities are observed to be unaffected in children with SLI. However, these studies did not rule out that other aspects of procedural learning and memory, such as difficulty in sequential auditory processing, could contribute to language problems in SLI. Checking whether the existence or absence of limitations holds for both visual and auditory modalities is also important because Ullman and Pierpont (2005) make the strong claim that their hypothesis implies the existence of procedural memory deficits in all modalities. As the idea of a deficit in nonverbal abilities is quite bold when it is associated to children with verbal knowledge deficits, it has received more attention in the literature. However, 
Gabriel et al.: Procedural learning in children with SLI

checking the existence of a procedural memory deficit in both modalities for the same children is important, as the existence of a different effect in the two modalities would have important consequences for the PDH.

In the domain of procedural learning, most research instantiates the SRT on the basis of visual events, and auditory stimuli have rarely been used (Frensch, Lin, \& Buchner, 1998). Some studies using auditory material have been conducted, but they were somewhat unsystematic in their approach to studying auditory sequence learning in its own right (Buchner, Steffens, Irmen, \& Wender, 1998; Saffran, Johnson, Aslin, \& Newport, 1999). It has been suggested that procedural learning processes also operate on auditory sequences. Buchner, Steffens, Erdfelder, and Rothkegel (1997) showed clear evidence of learning in a sequential tone discrimination task in undergraduate students. This result was confirmed by Saffran et al. (1999). In their experiment, participants were familiarized with a new language consisting of a three-tone sequence and were required to discriminate words (i.e., a learned sequence of tones) from nonwords. The results suggest that these tone stimuli were learned in a manner analogous to the speech stimuli both in adults (Experiment 2) and in 8-month-old infants (Experiment 3).

Sequence learning in SRT tasks has been investigated primarily with visual stimuli in SLI. No procedural learning SRT study in SLI used auditory information processing to investigate procedural learning abilities. Data are thus lacking regarding the different aspects of procedural learning in children with SLI. Such data could contribute to a better understanding of the conflicting results reported recently. This could also be the missing link between the nonverbal procedural learning abilities and the grammatical deficits observed in SLI children. We hypothesize that poorer procedural learning in SLI should be observed irrespective of whether the procedural memory is being tested in the auditory or visuospatial domain.

\section{AIMS}

The current study aims to compare the performance of children with SLI and TD peers on tasks that measure procedural learning both in auditory and visual modalities. In line with the proposal that implicit learning may involve multiple subsystems, which each handle different types of input (Conway \& Christiansen, 2006), some implicit learning systems (e.g., those handling auditory sequences) could be more impaired in SLI children than others.

To elucidate these issues, two versions of SRT tasks are proposed: a visual task and an auditory task. The current study offers an innovative exploration of visual and auditory sequential processing across SRT paradigms within the same sample of children with SLI. Its goal is to provide data about procedural sequence learning in SLI that differ from the usual data where both modalities are tested separately, at separate ages, using different methodologies.

\section{Methods}

Participants. Thirty-two children (16 children with SLI aged $119 \pm 23$ months and 16 TD children aged $118 \pm 22$ months, 15 boys in each group) participated in the study. No participant had previously taken part in any other implicit 
sequential learning study. Participants (range $=7-13$ years) were identified as having either normal learning development or SLI, with no other learning disorders. No child was diagnosed as having attention-deficit/hyperactivity disorder. TD children were recruited from schools near the University of Liège, Belgium. Children with SLI were recruited in a special educational setting for children with severe language disabilities, where they had received a previous clinical diagnosis of SLI by professionals (speech-language pathologists and child neurologists). All the children were Caucasian and came from families with a low or middle-class sociooccupational background, which was determined by their parents' profession (Institut national de la statistique et des études économiques, 2003). The social and occupational group of the child's family was defined on the basis of the head of the household's occupation. The three children (with or without SLI) from low socioeconomic status backgrounds were children whose parents were unemployed or homemakers (Category 8) and the thirteen children (with or without SLI) from middle socioeconomic status backgrounds were children with at least one parent who was a skilled or unskilled worker (Category 5: employed, Category 6: workers or agricultural laborers) but not managers (Category 3 ).

The parents were asked to complete a medical history questionnaire in order to ensure that all the children were French monolingual speakers, had no history of psychiatric or neurological disorders, and had no neurodevelopmental delay or sensory impairment (e.g., gross motor coordination disorder, visual impairment). The TD children presented no language impairment and no other, more general learning impairments. The parents of all children gave informed consent.

Children were tested individually in a quiet setting at their school. Each child with SLI was matched with a TD child based on socioeconomic status (i.e., matching was based on the level of education required to perform the parents' job), gender, Perceptual Reasoning Index ( \pm 8 points; Wechsler Intelligence Scale for Children, 4th ed., French version; Wechsler, 2005), and chronological age $( \pm 3$ months).

We applied diagnostic criteria for SLI in line with those typically used in studies of SLI in English-speaking children; that is, scores lower than or equal to $1.25 S D$ below the mean in two or more of four language tests in conjunction with performance IQ scores of 80 or higher (Wechsler Intelligence Scale for Children, 4th ed., French version; Wechsler, 2005). Perceptual Reasoning Index was calculated on the basis of three subtests (matrix reasoning, block design, and picture completion). We also administered a hearing test. All children had normal hearing. The criteria for normal hearing were the American Speech-LanguageHearing Association 1997 guidelines for hearing screening (at 500, 1000, 2000, and $4000 \mathrm{~Hz}$ and $20 \mathrm{~dB}$ ). A significant challenge for research with pediatric, language-impaired, French-speaking populations in Belgium is the scarcity of standardized tests to assist with identification. In order to identify children with SLI, a combination of referrals from speech pathologists and scores from nonstandardized as well as standardized tests was used. Thus, we administered a battery of standardized language tests to children with SLI in order to establish a profile of weaknesses for each child with SLI and to examine the relationships between SLI in French and procedural learning. The SLI group exhibited significant difficulties in producing and/or understanding language materials; specific difficulties were 
observed in phonology, grammar, and narrative. In order to allow the assessment of the PDH, children with SLI presented at least one grammatical deficit. Four language tests were administered: two receptive tests (Echelle de Vocabulaire en Images Peabody [EVIP]; Dunn, Thérault-Whalen, \& Dunn, 1993; Epreuve de Compréhension Syntaxico-Semantique [ECOSSE]; Lecocq, 1998) and two expressive tests (sentence production and word repetition, Evaluation du Langage Oral [ELO]; Khomsi, 2001). The EVIP (Dunn et al., 1993), which is a French adaptation of the Peabody Picture Vocabulary Test (Dunn \& Dunn, 1981), measures lexical knowledge. The ECOSSE (Lecocq, 1998), a French adaptation of the Test for Reception of Grammar (Bishop, 1989), measures receptive grammatical knowledge. We also administered two subtests of the Clinical Evaluation of Language (word repetition and sentence production) from the ELO battery (Khomsi, 2001). The word repetition task measures repetition performance for late-acquired phonemes, complex phonological patterns, and multisyllabic words. The sentence production task measures productive morphosyntactic abilities by assessing the children's ability to complete the sentences produced by the examiner.

TD children were administered the same tests as children with SLI, except for the sentence production component and word repetition in the ELO test battery. These children were reported to exhibit typical development in all areas assessed. Participant characteristics are reported in Table 1. At the beginning of the SRT task, participants were free to spontaneously choose one arm according to their hand preference. Once they had chosen their hand, the children were not allowed to use the other hand at any point during the task.

All children (with and without SLI) performed correctly at the end of the pretest for the SRT tasks across linguistic and nonlinguistic modalities. The tasks consisted of a series of 20 practice trials to test whether the children could use the auditory material of the SRT tasks.

No child was excluded from the study for reasons of major motor or visual impairment. We decided to exclude from the study two children with SLI (and their TD peers) because of their inability to use the auditory material of the auditory SRT task (see below). The statistical data were therefore calculated based on 28 children (14 children with SLI, 14 TD children) instead of 32 children ( 16 children with SLI, 16 TD children). We did not check whether the participants were righthanded or left-handed. The response panel used in our second SRT task required the children to press a button with either their right hand or their left hand according to their handedness. The local research ethics committee approved the study.

Stimulus materials and procedure. Control of the image presentation and recording speed of response and accuracy was performed using the E-Prime Software, Version 1.2. Participants were seated in front of the computer screen. The average eye/screen distance was $70 \mathrm{~cm}$. The SRT task was designed to make the task more attractive to children: the picture of a farm with four windows (i.e., the locations where the stimuli might appear) remained constantly displayed on a 15-in. personal computer screen (see Figure 1). Two windows were on the top floor of the farm (upper left and right) and two windows were on the ground floor (lower left and right). The distances between the two horizontal and vertical windows were 25 and $14.5 \mathrm{~cm}$, respectively. Children had to touch, as quickly and as accurately 
Table 1. Descriptive statistics for the administered measures

\begin{tabular}{|c|c|c|c|c|c|c|}
\hline & \multirow[b]{2}{*}{$\begin{array}{c}\text { Age } \\
\text { (months) }\end{array}$} & \multirow[b]{2}{*}{ PRI } & \multirow[b]{2}{*}{ EVIP } & \multirow[b]{2}{*}{ ECOSSE } & \multicolumn{2}{|c|}{ ELO } \\
\hline & & & & & $\begin{array}{c}\text { Word } \\
\text { Repetition }\end{array}$ & $\begin{array}{l}\text { Sentence } \\
\text { Production }\end{array}$ \\
\hline \multicolumn{7}{|c|}{ SLI ( 1 girl, 15 boys) } \\
\hline Mean & 119 & 99.8 & -0.64 & -1.39 & -24.65 & -5.48 \\
\hline$S D$ & 23 & 14.3 & 1.17 & 1.8 & 25.85 & 3.40 \\
\hline Range & $81-158$ & $81-140$ & -2.80 to 0.86 & -5.19 to 1.90 & -91.67 to -4.25 & -10.35 to -0.35 \\
\hline \multicolumn{7}{|c|}{ TD (1 girl, 15 boys) } \\
\hline Mean & 118 & 99.8 & 0.85 & -0.04 & NA & NA \\
\hline$S D$ & 22 & 13.2 & 0.78 & 1.33 & NA & NA \\
\hline Range & $83-156$ & $80-138$ & -0.33 to 2.33 & -4.52 to 1.41 & NA & NA \\
\hline$t$ for group diff. & $t(30)=-0.07$ & $t(30)=-0.00$ & $t(30)=4.24 * * *$ & $t(30)=3.39^{*}$ & NA & NA \\
\hline
\end{tabular}

Note: SLI, specific language impairment; TD, typically developing; PRI, Perceptive Reasoning Index; EVIP, French version of the Peabody Picture Vocabulary Test (Dunn \& Dunn, 1981), $Z$ scores with $M=0, S D=1$; Performance QI, block design, picture completion, and matrix subtests of the Wechsler Primary Scale of Intelligence-Revised (4th ed.), standard scores with $M=100, S D=15$; ECOSSE, French adaptation of the Test for Reception of Grammar (Bishop, 1989), $Z$ scores with $M=0, S D=1$ (minimum =0, maximum = 92); ELO, Evaluation du language oral (Khomsi, 2001), $Z$ scores with $M=0, S D=1$ (sentence production: minimum $=0$, maximum $=25$; word repetition: minimum 0 and maximum 32). This task measures repetition performance for late-acquired phonemes, complex phonological patterns, and multisyllabic words (Khomsi, 2001). The very poor word repetition performance observed in children with SLI is due to the lack of errors expected in older children. Whereas older TD children present a ceiling effect on a phonological task, older children with SLI continue to produce phonological mismatches. Therefore, the distance between the two groups increases, explaining otherwise seemingly incredible statistical scores. NA, not applicable. $* p<.05 . * * * p<.001$. 


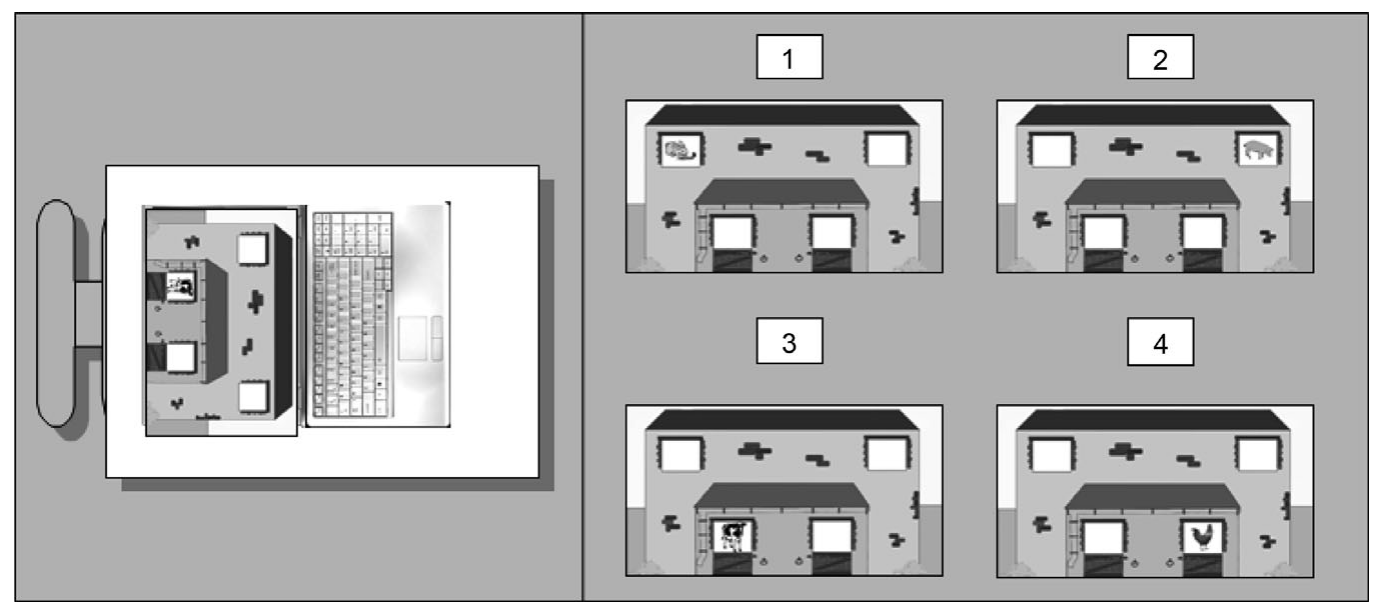

Figure 1. Schematic of computer display for the adapted serial reaction time (SRT) task (visual version) used in the visual SRT task. On each trial, an animal appeared at one of four possible locations (one of the four corner windows of the farm): Position 1 (upper left), Position 2 (upper right), Position 3 (lower left), and Position 4 (lower right). 
Gabriel et al.: Procedural learning in children with SLI

as possible, the location on the screen where the target appeared. As shown in Figure 1, the touch screen was placed on the laptop screen and was of the same size. The laptop screen was lowered so that the touch screen was at the same level as the keyboard (i.e., the angle between the keyboard and the laptop screen was $180^{\circ}$ ) and the picture of the scene was reversed. This position allowed the child to see the scene the right way up and to rest his or her elbow on the table, so that the situation was as comfortable as possible for the child. We deliberately chose this response mode because Gabriel, Stefaniak, Maillart, Schmitz, and Meulemans (in press) showed that when the SLI children had to respond by means of a touch screen, they responded as quickly and as accurately as their TD peers, while this was not the case for the same SRT task when using a computer keyboard as input device. It is as yet unclear why a touch screen offers such an advantage, but it appeared important to use an input method that was efficient for all children.

VISUAL SRT TASK. The experiment consisted of seven blocks of four-choice RT tasks. One experimental block consisted of an eight-element length sequence repeated eight times. Thus, each block involved 64 trials. There were six learning blocks (Block 1 to Block 6) and one transfer block (Block 7). The same eightelement length (1-3-4-2-3-1-2-4) was repeated from Block 1 to Block 6, making 384 learning trials altogether. Within the transfer block, another eight-element length sequence (4-2-1-3-2-4-3-1) was repeated eight times. Thus, there were 64 trials within the transfer block. In total, the children participated in 448 trials, divided up into seven blocks. In each trial, a stimulus (an animal) appeared in one of four possible locations (one of the four corner windows of a farm). The eightelement length sequence was an ambiguous sequence because each position could be followed by two different possible locations (Cohen, Ivry, \& Keele, 1990). In our experimental sequence 1-3-4-2-3-1-2-4, if 4 comes before 2, then 2 will be followed by 3 . However, 2 will follow 4 or 1 with a probability of 0.50 and it will follow 3 with a probability of 0 . Half of the participants were trained using the first ambiguous sequence (4-2-1-4-3-2-4-1) for Blocks 1 to 6 , with the second ambiguous sequence being used for Block 7 (the transfer block: 2-1-4-3-4-1-2-3). This design was reversed for the other half of the participants. Learning of the sequence in Blocks 1 to 6 may be attested by longer RTs in Block 7 than in Block 6 . Moreover, the visual stimulus appeared in each window on the computer screen an equal number of times for Blocks 1-6. The sequences were equated with respect to location frequency (each location occurred twice).

AUDITORY SRT TASK. In this version, we replaced the visual stimulus of the appearance of an owl in a drawing displayed on the screen with an auditory stimulus (the screech of an owl) that the child had to locate in space (left vs. right, far vs. near). The children had to associate the location of the sound in space with a location in a drawing displayed on the screen. So the children had to made this correspondence themselves, whereas in the visual stimuli they had to point at the very location where a stimulus appeared. Note that this change introduces substantial modifications in the way the task is introspectively apprehended by the participants, because response stimulus mapping, which is direct in the visual 
procedure, requires some controlled intermediary processes in the case of sounds. In order to create an experimental situation that was intuitive and simple enough to be used by young children, especially children with SLI, we chose to use a difference between sounds reduced to two opposite values only: amplitude intensity (high vs. low). A second dimension (left vs. right) was added, which meant that altogether four values were coded. In this SRT task, children had to touch the location of the sound as quickly and as accurately as possible. For the sequence learning task, the stimuli were brief, computer synthesized sounds (the screech of an owl) played binaurally through stereo headphones that were plugged into the computer. Four different sounds were used with the same frequencies $(11025 \mathrm{~Hz}$ ) but with different intensities (loudest sound, $85 \mathrm{~dB}$, vs. lowest sound, $55 \mathrm{~dB}$ ) and presented either in the right or in the left ear. Each sound was assigned to a location on the touch screen.

A pretest was achieved to check whether children could discriminate among the four types of sounds (left vs. right, high intensity vs. low intensity). No significant statistical difference between the two groups concerning the number of correct responses during the pretest of the auditory SRT task was observed, $F(1,26)=$ $0.29, M S E=11846, p=.59, \eta_{\mathrm{p}}^{2}=0.011$. Moreover, the interaction was not found to be significant, $F(3,78)=0.08, M S E=40261, p=.49, \eta_{\mathrm{p}}^{2}=0.030$, suggesting that the four practice conditions were similar in both groups. The mean of the median response RTs for correct responses was calculated for each block, as is common practice in studies using an SRT task (Nissen \& Bullemer, 1987). We performed an analysis of variance (ANOVA) with Types of sounds (left vs. right, high intensity vs. low intensity) as a within-participant variable, and Group (two levels: TD vs. SLI) as a between-participant variable. No significant statistical difference between the two groups was observed, $F(1,26)=1.43, M S E=0.057$, $p=.24, \eta_{\mathrm{p}}^{2}=0.052$. Moreover, the interaction was not found to be significant, $F(3,78)=1.89, M S E=0.01021, p=.14, \eta_{\mathrm{p}}^{2}=0.067$, suggesting that the difference between the four types of sounds were similar in both groups. Two children were excluded from this task because they were characterized as outliers who presented RTs that were $2 S D$ from the mean of the SLI group.

Low (high) sounds were symbolized by far (near) trees within the pictures while the child had to choose the right (left) tree for sounds presented in the right (left) ear (i.e., a low left sound for the upper left, a low right sound for the upper right, a high left sound for the lower left and, a high right sound for the lower right). Two different eight-trial sequences were used, both of which were ambiguous sequences according to the distinction introduced by Cohen et al. (1990). We constructed the first sequence by assigning Sound 1 to A, Sound 2 to B, Sound 3 to C, and Sound 4 to D. With respect to Sounds 1 to 4 , this first sequence can be characterized as C-A-B-D-A-C-D-B. Sequence 2 was A-B-D-C-D-B-A-C. During this task, the participants were instructed to touch the location of the stimulus (a sound) on one of four trees inside the wood as quickly and as accurately as possible after hearing a sound (see Figure 2a). The sequences used differed from those in the visual SRT task, but these sequences were designed to introduce the same level of complexity. Half of the participants were trained with the first sequence for Blocks 1 to 6 , and with a second sequence for Block 7; this design was reversed for the other half 
Gabriel et al.: Procedural learning in children with SLI

(a)

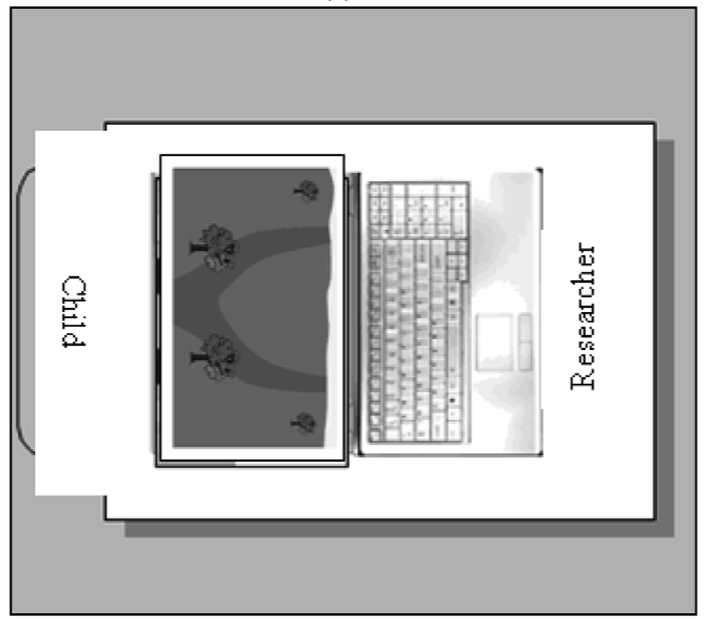

(b)

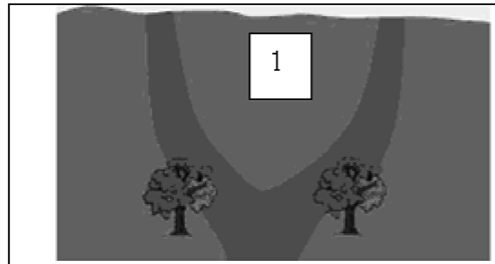

First oondition: high right soumd vs. high left soumd.

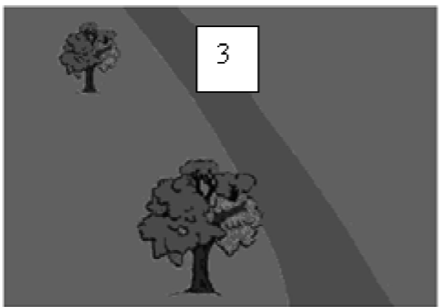

Third oondition: low left sound $v$ s. high bft sound.

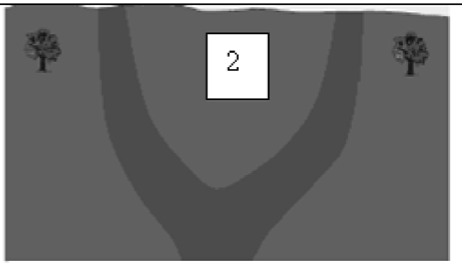

Second oondition: bw right sound vs. bw left sound

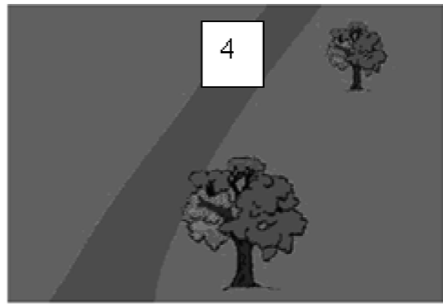

Fourth oondition: low right soumd vs. high rijht sound.

Figure 2. Schematic of computer display for the adapted serial reaction time (SRT) task (auditory version) used in the auditory SRT task. (a) Four different sounds were used with the same frequencies but with different intensities (high vs. low). Each sound was assigned to a location on the touch screen (one of the four trees inside the wood): Position 1 (low left), Position 2 (low right), Position 3 (high left), and Position 4 (high right). (b) The task began with a series of 20 randomly generated practice trials for each condition: first condition (high right sound vs. high left sound), second condition (low right sound vs. low left sound), third condition (low left sound vs. high left sound), fourth condition (low right sound vs. high right sound). 
Gabriel et al.: Procedural learning in children with SLI

of the participants. Participants were not informed of the presence of a sequence. The task began with a pretest that consisted of a series of 20 randomly generated practice trials for each condition (first condition: high right sound vs. high left sound, second condition: low right sound vs. low left sound, third condition: low left sound vs. high left sound, fourth condition: low right sound vs. high right sound; see Figure 2b).

Children were administered these two SRT tasks in two sessions lasting approximately $20 \mathrm{~min}$. Half of the participants began with the visual task, followed by the auditory task; this design was reversed for the other half of the participants in order to rule out an order effect during administration of the tasks. A delay of 6 weeks separated the two tasks.

\section{Results}

A student $t$ test was conducted in order to rule out an order effect during administration of the tasks. No significant statistical difference was observed between the order effects during task administration for visual learning indexes (Block 7 Block 6$) /($ Block $6+$ Block 7$), t(1,26)=1.07, p=.29, \eta_{\mathrm{p}}^{2}=0.18$, and for auditory learning indexes, $t(1,26)=0.04, \mathrm{p}=.96, \eta_{\mathrm{p}}^{2}=0.02$. No significant difference was observed between sequence type during task administration for visual and for auditory conditions.

$R T$ analyses. We first performed an ANOVA with Modality (two levels: visual vs. auditory) and Blocks (six levels: Block 1 to Block 6) as within-participant variables, and Group (two levels: TD vs. SLI) as a between-participant variable. The results showed that the RTs of children with SLI were similar to those of TD children, $F(1,26)=0.47, M S E=299191, p=.49, \eta_{\mathrm{p}}^{2}=0.017$, that the RT decrease from Block 1 to Block 6 was significant, $F(5,130)=19.18, M S E=$ $11225, p<.001, \eta_{\mathrm{p}}^{2}=0.42$, and that this decrease was similar for both groups, as shown by the nonsignificant interaction, $F(5,130)=0.40, M S E=11225, p=.84$, $\eta_{\mathrm{p}}^{2}=0.015$. Results also showed that visual stimuli were processed more quickly than auditory stimuli, $F(1,26)=60.28, M S E=194374, p<.001, \eta_{\mathrm{p}}^{2}=0.70$, in both groups as shown by the nonsignificant interaction, $F(1,26)=0.06, M S E=$ 194374, $p=.79, \eta_{\mathrm{p}}^{2}=0.002$. The other interactions did not reach significance.

Because learning is considered to be sequence specific when RTs slow down between the last learning block (i.e., Block 6) and the transfer block (i.e., Block 7), we performed an ANOVA with Modality (two levels: visual vs. auditory) and Block (two levels: Block 6 vs. Block 7) as within-participant variables, and Group (two levels: TD vs. SLI) as a between-participant variable. This analysis once again found that children with SLI were not significantly slower than their TD peers, $F(1,26)=0.84, M S E=68686, p=.36, \eta_{\mathrm{p}}^{2}=0.031$, and also showed that Block 6 was processed faster than Block 7, $F(1,26)=41.99, M S E=20402$, $p<.001, \eta_{\mathrm{p}}^{2} 0.62$, and that this difference was similar for both groups, as shown by the nonsignificant interaction, $F(1,26)=1.07, M S E=20402, p=.31$, $\eta_{\mathrm{p}}^{2}=0.039$. (See Figure 3 for all RT results.) We also performed separate analyses for visual and auditory modalities to rule out the possibility of insufficient power in 
Gabriel et al.: Procedural learning in children with SLI
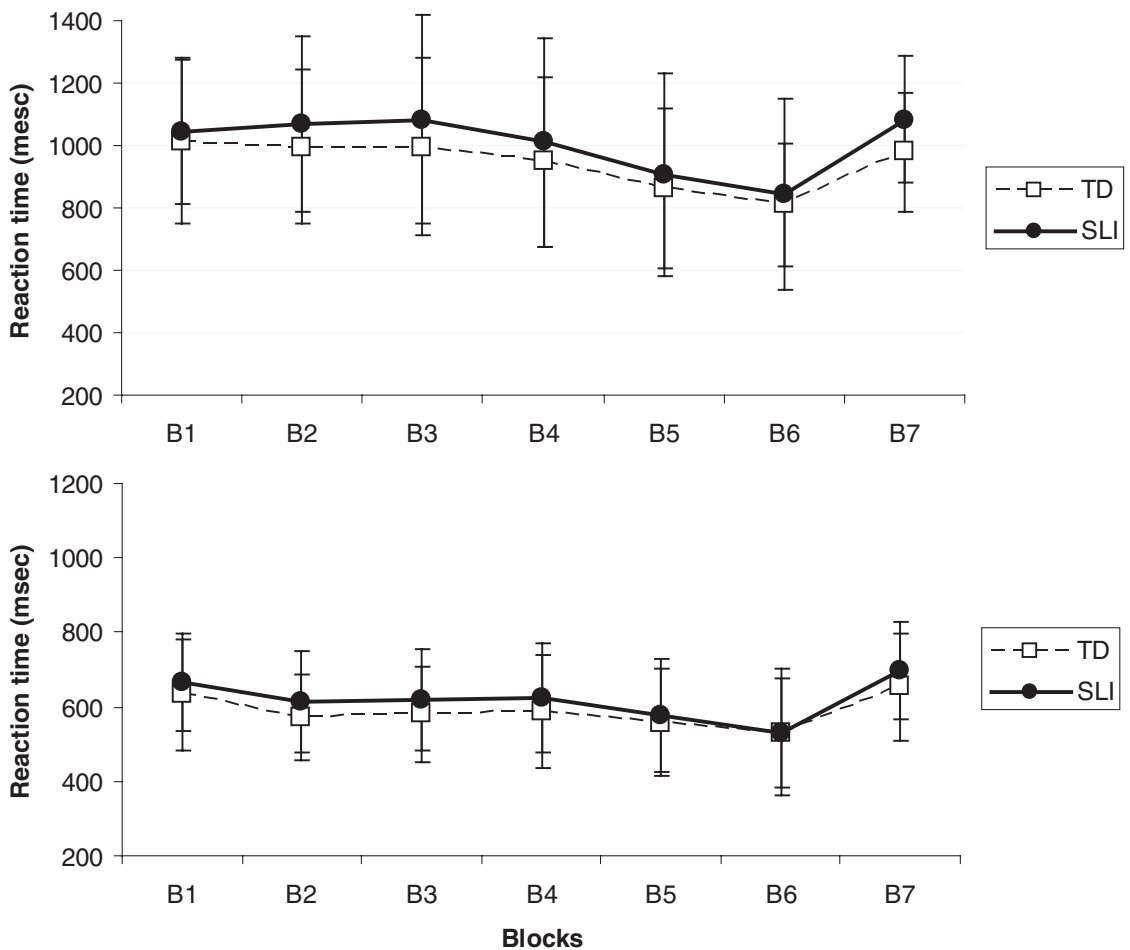

Figure 3. Mean reaction times (RTs) for each block for children with specific language impairment (SLI) and typically developing (TD) children during the adapted SRT tasks. Blocks 1-6: structured; Block 7: random. Learning is indicated by the RT increase in the random Block 7 compared to the preceding and subsequent structured Blocks 6, respectively. Bars represent standard deviations of the mean.

our data. We first performed an ANOVA in the auditory modality with Block (two levels: Block 6 vs. Block 7) as a within-participant variable, and Group (two levels: TD vs. SLI) as a between-participant variable. The results showed no group effect, $F(1,26)=0.75, p=.39, \eta_{\mathrm{p}}^{2}=0.028$, a Block effect, $F(1,26)=33.63, p<.001$, $\eta_{\mathrm{p}}^{2}=0.56$, and no interaction effect, $F(1,26)=1.05, p=.31, \eta_{\mathrm{p}}^{2}=0.039$. We then performed the same analysis in the visual modality and found comparable results: no group effect, $F(1,26)=0.21, p=.64, \eta_{\mathrm{p}}^{2}=0.008$, a Block effect, $F(1,26)=25.12 ; \mathrm{p}<.001, \eta_{\mathrm{p}}^{2}=0.49$, and no interaction effect, $F(1,26)=$ $0.46, p=.503, \eta_{\mathrm{p}}^{2}=0.017$. Finally, results also showed that visual stimuli were processed more quickly than auditory stimuli, $F(1,26)=57.2, M S E=51824$, $p<.001, \eta_{\mathrm{p}}^{2}=0.068$, in both groups as shown by the nonsignificant interaction, $F(1,26)=0.29, M S E=51824, p=.59, \eta_{\mathrm{p}}^{2}=0.011$. The other interactions did not reach significance.

We computed a learning index for each participant. This index corresponds to the value (Block $7-$ Block 6)/(Block $6+$ Block 7$)$. A value superior to 0 indicates 
Gabriel et al.: Procedural learning in children with SLI

that some learning took place during the experiment (Meulemans et al., 1998; Thomas \& Nelson, 2001). The mean for children with SLI was $0.14(S D=0.15)$ for the visual condition and $0.15(S D=0.16)$ for the auditory condition. The mean for TD children was $0.11(S D=0.10)$ for the visual condition and $0.10(S D=$ 0.08 ) for the auditory condition. A $t$ test showed that the learning indices were similar in both groups, $t(14)=-0.64, p=.53$ (visual condition) and $t(14)=-1.03$, $p=.31$ (auditory condition). Moreover, single-sample $t$ tests (two-tailed) indicated that the TD group obtained an average learning index that was significantly greater than zero, $t(14)=3.95, p<.001$ (visual condition) and $t(14)=4.37, p<.001$ (auditory condition). Similarly, the SLI group obtained an average learning index that was significantly greater than zero, $t(14)=3.47, p=.004$ (visual condition) and $t(14)=3.51, p=.003$ (auditory condition). This indicates that both the TD group and the SLI group demonstrated significant sequence-specific learning in both modalities.

Analyses of the correctness of response. In order to ensure that the absence of difference between the RT decreases observed in both groups was not related to differences in accuracy, we checked whether the accuracy changed during the experiment. We conducted an ANOVA with Modality (two levels: visual vs. auditory) and Blocks (two levels: Block 6 vs. Block 7) as within-participant variables, and Group (two levels: TD vs. SLI) as a between-participant variable on the logarithms of correct responses. The analysis revealed that children with SLI made fewer correct responses than TD children, $F(1,26)=9.34, M S E=0.01116, p=.005, \eta_{\mathrm{p}}^{2}=$ 0.26 . There was no difference between the last learning block (Block 6) and the transfer block (Block 7), $F(1,26)=3.90, M S E=0.0007, p=.06, \eta_{\mathrm{p}}^{2}=0.13$, and this was similar in both groups, as shown by the nonsignificant interaction, $F(1,26)=1.90, M S E=0.0007, p=.18, \eta_{\mathrm{p}}^{2}=0.068$. These results show that children with SLI made fewer correct responses than controls but also that this difference was stable between Block 6 and Block 7 for each group. A significant Modality effect was also observed, $F(1,26)=19.07, M S E=0.0125, p<.001, \eta_{\mathrm{p}}^{2}$ $=0.42$. Moreover, the Modality $\times$ Group interaction was significant, $F(1,26) \stackrel{=}{=}$ $8.05, M S E=0.0125, p=.008, \eta_{\mathrm{p}}^{2}=0.23$. Planned comparison showed that children with SLI, contrary to TD children, made more errors in the auditory SRT task compared to the visual SRT task, $F(1,26)=25.94, p<.001$. Figure 4 shows the mean proportion of correct responses for both Block 6 and 7 plotted separately for each group. The triple interaction, Blocks $\times$ Modality $\times$ Group, was not significant, $F(1,26)=0.91, M S E=0.00009, p=.35, \eta_{\mathrm{p}}^{2}=0.003$.

A supplementary analysis was done to compare the number of correct responses between the first learning block (Block 1) and the last learning block (Block 6) in children with SLI. The rate of correct responses was stable, $F(1,26)=2.50$, $M S E=0.0019, p=.12, \eta_{\mathrm{p}}^{2}=0.08$.

Reaction time and vocabulary or grammar status. As in Lum et al.'s study (2012), associations between the procedural memory and language variables were examined with correlations (Pearson $r$ ) computed for each language-ability measure, separately for TD and SLI groups. For procedural memory, we used the $z$ score of SRT learning indices (Block 6-Block 7)/(Block 6 + Block 7). For lexical abilities, 
Gabriel et al.: Procedural learning in children with SLI
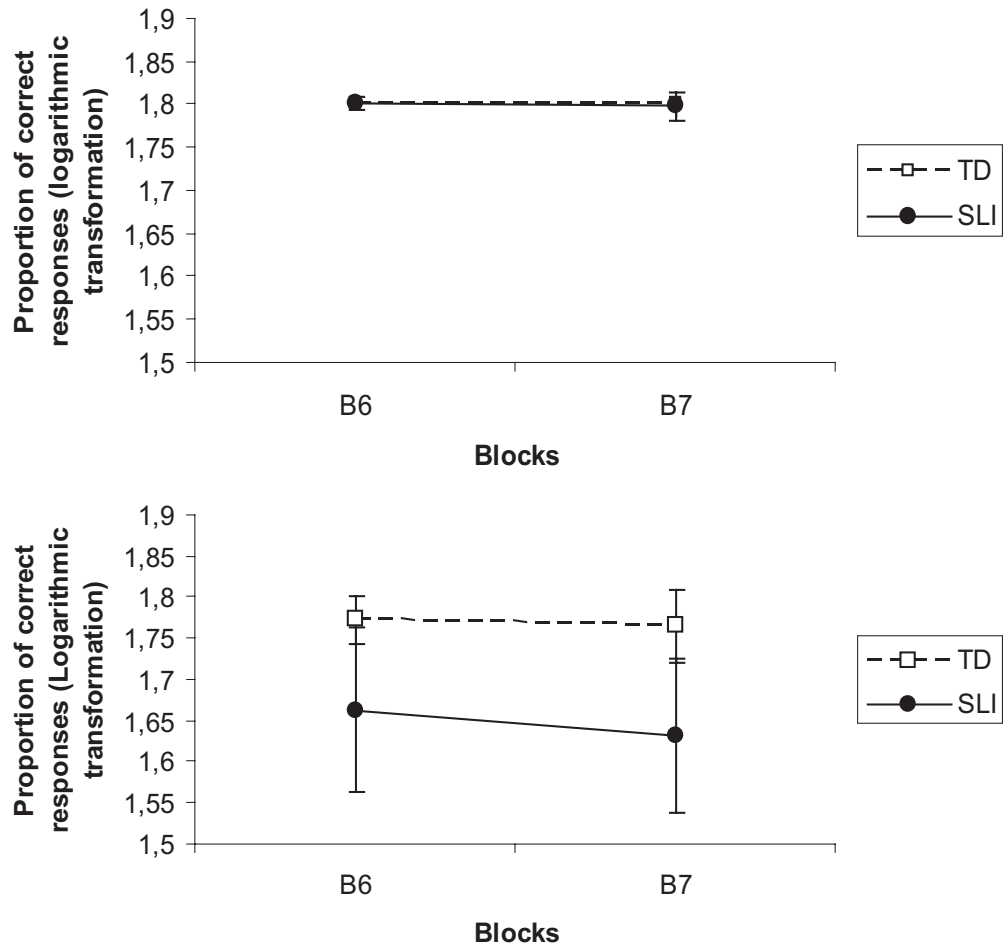

Figure 4. Correct responses for children with specific language impairment (SLI) and typically developing (TD) children during the adapted serial reaction time tasks. Bars represent standard deviations of the mean.

we used the $z$ score of the receptive (EVIP) test. For grammatical abilities, we used the $z$ score of the expressive (ELO: sentences production) test, and the $z$ score of the receptive (ECOSSE) grammar tests.

Regarding SLI participants, correlation analyses revealed that learning scores in the auditory SRT task did not correlate with the scores in grammar knowledge (ECOSSE: $r=-.17, p=.56$; ELO: $r=.12, p=.69$ ) nor with the scores in lexical knowledge (EVIP: $r=.01, p=.96$ ). Learning in the auditory SRT task did not correlate with phonological abilities in children with SLI (ELO: word repetition: $r=.29, p=.32$ ). Similarly, learning in the visual SRT task did not correlate with the scores in grammar knowledge (ECOSSE: $r=-.46, p=.11$; ELO: $r=.15$, $p=.61$ ) nor with the scores in lexical knowledge (EVIP: $r=-.03, p=.91$ ).

Regarding TD participants, correlation analyses revealed that learning in the auditory SRT task did not correlate with the grammar knowledge (ECOSSE: $r=$ $-.32, p=.25$ ) nor with the lexical knowledge (EVIP: $r=-.12, p=.67$ ). Similarly, learning in the visual SRT task did not correlate with the performance in grammar knowledge (ECOSSE: $r=-.28, p=.32$ ) nor with the performance in lexical knowledge (EVIP: $r=-.39, p=.16$ ). 
Gabriel et al.: Procedural learning in children with SLI

Overall, our results did not demonstrate that low grammar abilities may be directly related with low performance in procedural learning in SRT tasks, as suggested by the PDH (Ullman \& Pierpont, 2005). In the current study, neither receptive grammatical abilities nor expressive grammatical abilities were associated with procedural memory in children with SLI. However, given the small sample, the correlation between grammatical and lexical abilities with SRT learning indices must be treated with caution.

\section{DISCUSSION}

Deficits in SLI have been associated with a deficit in procedural processing (Ullman \& Pierpont, 2005). However, although most procedural studies support the predictions of the procedural deficit hypothesis in SLI (Adi-Japha et al., 2011; Evans et al., 2009; Hsu et al., 2006; Kemény \& Lukács, 2010; Lum et al., 2010; Lum et al., 2012; Plante et al., 2002; Tomblin et al., 2007), recent studies (Gabriel et al., 2011; Hedenius et al., 2011; Lum \& Bleses, 2012) also revealed that children with SLI were able to learn implicit sequential data from visual input.

The aim of this study was to contribute to the current understanding of the nature of the procedural deficit by examining whether SLI children differ from TD children in the procedural learning of sequential information from auditory input and from visual input.

To investigate this question, we administrated two SRT tasks in children with SLI: a visual SRT task and an auditory one. Results of the visual SRT task showed that children with SLI were as fast and as accurate as their TD peers. Furthermore, they presented similar specific sequence learning indices. These findings concur with recent SRT studies (Gabriel et al., 2011; Hedenius et al., 2011; Lum \& Bleses, 2012) reporting that children with SLI did not differ from controls in the procedural learning of sequential information from visual input. Despite expectations to the contrary, children with SLI did not differ from their TD peers in the procedural learning of sequential information from auditory input, at least for the speed and for the learning indices. Our findings therefore suggest that procedural memory may not be highly stimuli-dependent since they show that children with SLI were able to learn sequential information, regardless of the type of information that was manipulated. An important issue arising from these data concerns the extent to which procedural learning in SLI is partially supported by a domain-general learning mechanism rather than by a stimulus-specific mechanism.

Various reasons can explain the difference between our results and previous results, such as those of Tomblin et al. (2007) or Lum et al. (2010). Many studies suggest that orally mediated sequence learning under statistical learning conditions may be impaired in SLI, whereas visually mediated sequence learning can present contrasting results: some studies observed preserved capacities while others reported impaired learning. Therefore, visually mediated sequence learning in SLI could be spared under some conditions. Evans et al. (2009) also showed that children with SLI were able to track transitional probabilities in the speech condition with increasing input. Tomblin et al. (2007) also found that adolescents with grammar impairment required significantly more trials to learn sequential elements in their SRT task than did their grammar-normal peers. Moreover, the 
statistical structure of the SRT sequence differed from one study to another. The nature of the response equipment used (keyboard, gamepad, button box, or touch screen), seemed to have a considerable impact, as demonstrated by Gabriel et al. (2011, in press). These results suggest that a variety of factors related to the nature of the stimuli and equipment used in the experiments may better account for the performance of the children with SLI than the hypothesized deficit in detection of regularities. This could imply that children with SLI have access to procedural memory, including in the auditory modality, but that their abilities are not up to the level of those of TD children.

The results of the auditory SRT task also require careful consideration: a dissociation between preserved visual processing and impaired auditory processing was observed on the error rate. Children with SLI made more errors than their TD peers. Thus, the decrease in RTs associated with a lower percentage of correct responses in children with SLI calls into question the presence of a specific sequence learning effect during the auditory SRT task.

However, the error rate did not prove to be a relevant measure to assess SRT. The error rate between the first learning block (Block 1) and the last learning block (Block 6) in children with SLI was stable, as it was for TD children. This means that there was no learning effect on the error rate, including for TD children. The error rate was higher in children with SLI right from the beginning of the task (learning blocks), and it could therefore not be attributed to a tiredness effect or to attention fluctuations, which were more marked in the SLI group than in the TD group. An inhibition deficit (Botting, 2005; Marton, Kelmenson, \& Pinkhasova, 2007) cannot explain this increase in errors either, as children with SLI did not make more errors during the visual SRT task. Moreover, the error rate of children with SLI was higher on Block 7 than for all other blocks. Although not significant in the present study, this effect might have been significant with more statistical power. This would demonstrate that TD children and children with SLI differ with regard to the error rate in SRT tasks but only in the auditory modality. This means that the error rate might not be related to a deficit in procedural learning but to a deficit in auditory processing in general.

This leads to the conclusion that poor procedural learning does not necessarily indicate that this is the core deficit in SLI. Children with SLI may produce more errors than TD peers during the auditory SRT task because the incoming information is not adequately perceived, implying that the domain-general learning mechanism is not impaired in SLI. A procedural learning deficit could arise as the downstream consequence of an associated nonlinguistic deficit (e.g., a limitation in perception).

We hypothesized that children with SLI would have difficulties with sustained selective attention for auditory information (Spaulding, Plante, \& Vance, 2008). The result of this study showed that children with SLI performed less accurately than did controls for the auditory stimuli while there were no significant group differences for visual stimuli. During the auditory pretest, however, there was no difference between the two groups. These findings suggest that, while the predictions remain to be tested in more detail, a sustained selective attention deficit for auditory information may explain, at least in part, the difficulties children with SLIs encounter in tasks as the processing load increases, which is the case with with procedural tasks (Ellis Weismer \& Hesketh, 1996). Our future research should take the attentional capacities of participants into consideration. 
Children with SLI may make more errors than their TD peers during the auditory SRT task. The correlation analysis revealed that learning in the auditory SRT task did not correlate with phonological abilities in children with SLI, so limitations in the auditory modality could not be explained by insufficient phonological knowledge. A possible explanation could be that, as suggested by Hsu and Bishop (2010), the children's performance could be linked to perceptive limitations rather than to a procedural deficit.

Our study, as well as most previous studies, does not rule out the possibility that other aspects of procedural learning may be dysfunctional in SLI, for example, as demonstrated by Hedenius et al. (2011), long-term retention of procedural knowledge. Hedenius et al. (2011) suggest that the initial regularities extraction of procedural learning may be unimpaired but that consolidation and longer term procedural learning may cause the children more problems, which was not tested here. These findings are broadly consistent with Adi-Japha et al. (2011) regarding the consolidation of procedural learning in SLI. Adi-Japha et al. showed consolidation gains in TD children $24 \mathrm{hr}$ after training but not in children with SLI. Both Hedenius et al. and Adi-Japha et al. showed that, over time, children with SLI tend to forget what they have learned. Other explanations might be that we do not fully understand what is implied in procedural learning and the link with learning linguistic knowledge. For example, we did not fully test whether children used implicit learning or whether some explicit learning took place. If explicit learning took place, as is possible in SRT experiments (Howard et al., 2004; Howard \& Howard, 1997), then the SLI children could have used some compensatory mechanism. Dominey, Hoen, Blanc, and Lelekov-Boissard (2003) have shown that individuals with impaired syntactic abilities (e.g., left hemisphere aphasia) can learn serial structure but are not capable of extracting abstract structure on SRT tasks. This could have been a factor in our study.

To assess the PDH in SLI, we investigated whether individual differences in SRT task learning were more strongly associated with individual differences in grammar abilities than in lexical abilities, as suggested by the PDH. We did not find any correlation between receptive and expressive grammatical knowledge and visual or auditory SRT learning indices. These findings were thus not consistent with the proposal linking low grammar abilities to impairment in procedural memory. The study by Lum and Bleses (2012) provided similar data by reporting the absence of a link between poor expressive grammar abilities in children with SLI and their procedural abilities.

This study is innovative because it provides the first comparison of SRT learning in SLI across visual and auditory modalities using a within-subject design. The advantage of such a design is that interindividual variance is held constant, unlike in previous studies that compared only visual sequence learning between different individuals participating in different experiments.

\section{CONCLUSION}

The current study does not support the existence of a deficit in the extraction of regularities in children with SLI. Nevertheless, our results are valid for initial learning and cannot be generalized to later consolidation processes. Both the 
SLI and TD groups were comparable on procedural tasks involving nonlinguistic stimuli (visual vs. auditory). We therefore argue that the core of the impairment of SLI might not be linked to difficulty in learning nonlinguistic regularities. In fact, these unexpected data show that children with SLI were able to discern certain nonlinguistic regularities in the input to the same extent as TD children but that some weaknesses may emerge depending on the nature of the stimuli. However, these weaknesses cannot account alone for the mixed reports of sequence learning performance in SLI. As the results reported in the literature regarding regularities extraction in children with SLI are still limited, this is, for the moment, an open research question with important consequences for both SLI and procedural learning. The deficit in the extraction of regularities does not seem to be a sufficient cause to account for linguistic and nonlinguistic deficits in children with SLI. Future studies will be needed to identify alternative causes for these deficits. According to Bishop (2006, p. 1166), "a single cause approach is too simple to account for clinical reality." For example, our findings reflect weaknesses in auditory processing in children with SLI. When combined with other risk factors or other underlying deficits, these weaknesses could lead to different comorbidity profiles.

\section{ACKNOWLEDGMENTS}

The support for the conduct of this research was provided by the University of Liège (doctoral fellowship for fields not eligible for FRIA). We also thank Leslie Fauconnier, the teachers, children, and families who generously contributed their time.

\section{REFERENCES}

Adi-Japha, E., Strulovich-Schwartz, O., \& Julius, M. (2011). Delayed motor skill acquisition in children with language impairment. Research in Developmental Disabilities, 32, 2963-2971.

Archibald, L. M., \& Gathercole, S. E. (2007). The complexities of complex memory span: Storage and processing deficits in specific language impairment. Journal of Memory and Language, 57, 177-194.

Bishop, D. V. M. (1989). Test for Reception of Grammar. Manchester: University of Manchester, Age and Cognitive Performance Research Centre.

Bishop, D. V. M. (2002). Motor immaturity and specific speech and language impairment: Evidence for a common genetic basis. American Journal of Medical Genetics (Neuropsychiatric Genetics), $114,56-63$.

Bishop, D. V. M. (2006). Developmental cognitive genetics: How psychology can inform genetics and vice versa. Quarterly Journal of Experimental Psychology, 59, 1153-1168.

Bishop, D. V. M., Adams, C. V., Nation, K. \& Rosen, S. (2005). Perception of transient nonspeech stimuli is normal in specific language impairment: Evidence from glide discrimination. Applied Psycholinguistics, 26, 175-194.

Bishop, D. V. M., \& Norbury, C. F. (2005). Executive functions in children with communication impairments, in relation to autistic symptomatology: 2. Response inhibition. Autism, 9, $29-43$.

Botting, N. (2005). Non-verbal cognitive development and language impairment. Journal of Child Psychology and Psychiatry, 46, 317-326.

Buchner, A., Steffens, M., Erdfelder, E. \& Rothkegel, R. (1997). A multinominal model to assess fluency and recollection in a sequence learning task. Quarterly Journal of Experimental Psychology, 50A, 631-663.

Buchner, A., Steffens, M., Irmen, L., \& Wender, K. (1998). Irrelevant auditory material affects counting. Journal of Experimental Psychology: Learning, Memory, and Cognition, 24, 48-67. 
Gabriel et al.: Procedural learning in children with SLI

Campbell, W. N., \& Skarakis-Doyle, E. (2007). School-aged children with SLI: The ICF as a framework for collaborative service delivery. Journal of Communication Disorders, 40, 513-535.

Cohen, A., Ivry, R. I., \& Keele, S. W. (1990). Attention and structure in sequence learning. Journal of Experimental Psychology: Learning, Memory, and Cognition, 16, 17-30.

Cohen, N. J., \& Squire, L. R. (1980). Preserved learning and retention of pattern analysing skill in amnesia: Dissociation of knowing how and knowing that. Science, 210, 207-210.

Conway, C. M., \& Christiansen, M. H. (2006). Statistical learning within and between modalities: Pitting abstract against stimulus specific representations. Psychological Science, 17, 905-912.

Dominey, P. F., Hoen, M., Blanc, J. M., \& Lelekov-Boissard, T. (2003). Neurological basis of language and sequential cognition: Evidence from simulation, aphasia, and ERP studies. Brain and Language, 86, 207-225.

Doyon, J., Korman, M., Morin, A., Dostie, V., Hadj Tahar, A., Benali, H., et al. (2009). Contribution of night and day sleep vs. simple passage of time to the consolidation of motor sequence and visuomotor adaptation learning. Experimental Brain Research, 195, 15-26.

Dunn, L. M., \& Dunn, L. M. (1981). Peabody Picture Vocabulary Test, revised: Manual for forms L and $M$. Circle Pines, MN: American Guidance Service.

Dunn, L. M., Thériault-Whalen, C. M., \& Dunn, L. M. (1993). Echelle de vocabulaire en images Peabody: Adaptation française du Peabody Picture Vocabulary Test. Toronto: Psycan.

Eichenbaum, H. (2000). A cortical-hippocampal system for declarative memory. Nature Reviews Neuroscience, 1, 41-50.

Ellis Weismer, S., \& Hesketh, L. J. (1996). Lexical learning by children with specific language impairment: Effects of linguistic input presented at varying speaking rates. Journal of Speech and Hearing Research, 39, 177-190.

Ellis Weismer, S., Plante, E., Jones, M., \& Tomblin, J. B. (2005). A functional magnetic resonance imaging investigation of verbal working memory in adolescents with specific language impairment. Journal of Speech, Language, and Hearing Research, 48, 405-425.

Evans, J., Saffran, J. R., \& Robe-Torres, K. (2009). Statistical learning in children with specific language impairment. Journal of Speech, Language, and Hearing Research, 52, 321335.

Fernell, E., Norrelgen, F., Bozkurt, I., Hellberg, G., \& Löwing, K. (2002). Developmental profiles and auditory perception in 25 children attending special preschools for language-impaired children. Acta Paediatrica, 91, 1108-1115.

Frensch, P., Lin, J., \& Buchner, A. (1998). Learning versus behavioral expression of the learned: The effects of a secondary tone-counting task on implicit learning in the serial reaction task. Psychology Research, 61, 83-98.

Fromm, W., Schöler, H., \& Scherer, C. (1998). Jedes vierte Kind sprachgestört? Definition, Verbreitung, Erscheinungsbild, Entwicklungsbedingungen und-voraussetzungen der Spezifischen Sprachentwicklungsstörung. In H. Schöler, W. Fromm, \& W. Kany (Eds.), Spezifische Sprachentwicklungsstörung und Sprachlernen (pp. 22-63). Heidelberg: Winter.

Gabriel, A., Maillart, C., Guillaume, M., Stefaniak, N., \& Meulemans, T. (2011). Exploration of serial structure procedural learning in children with language impairment. Journal of the International Neuropsychological Society, 17, 1-8 .

Gabriel, A., Stefaniak, N., Maillart, C., Schmitz, X., \& Meulemans, T. (in press). Procedural visual learning abilities in children with specific language impairment. American Journal of Speech Language Pathology. doi:10.1044/1058-0360

Gabrieli, J. D. E (1998). Cognitive neuroscience of human memory. Annual Review of Psychology, 49, 87-115.

Hedenius, M., Persson, J., Tremblay, A., Adi-Japha, E., Verissimo, J., Dye, C. D., et al. (2011). Grammar predicts procedural learning and consolidation deficits in children with specific language impairment. Research in Developmental Disabilities, 32, 2362-2375.

Hill, E. L. (2001). Non-specific nature of specific language impairment: A review of the literature with regard to concomitant motor impairments. International Journal of Language \& Communication Disorders, 36, 149-171.

Hill, E. L., Bishop, D. V. M., \& Nimmo-Smith, I. (1998). Representational gestures in developmental coordination disorder and specific language impairment: Error-types and the reliability of ratings. Human Movement Science, 17, 655-678. 
Gabriel et al.: Procedural learning in children with SLI

Hoffman, L. M., \& Gillam, R. B. (2004). Verbal and spatial information processing constraints in children with specific language impairment. Journal of Speech, Language, and Hearing Research, $47,114-125$.

Howard, D. V., Howard, J. H., Jr., Japikse, K. C., DiYani, C., Thompson, A., \& Somberg, R. (2004). Implicit sequence learning: Effects of level of structure, adult age, and extended practice. Psychology and Aging, 19, 79-92.

Howard, J. H., Jr., \& Howard, D. V. (1997). Age differences in implicit learning of higher order dependencies in serial patterns. Psychology and Aging, 12, 634-656.

Hsu, H-J., \& Bishop, D. V. M (2010). Grammatical difficulties in children with specific language impairment: Is learning deficient? Human Development, 53, 264-277.

Hsu, H-J., Christiansen, M. H., Tomblin, J. B., Zhang, X., \& Gomez, R. L. (2006). Statistical learning of nonadjacent dependencies in adolescents with and without language impairment. Poster session presented at the 2006 Symposium on Research in Child Language Disorders, Madison, WI.

Im-Bolter, N., Johnson, J., \& Pascual-Leone, J. (2006). Processing limitations in children with specific language impairment: The role of executive function. Child Development, 77, 18221841 .

Institut national de la statistique et des études économiques. (2003). Insee-Réunion. Paris: INSEE.

Jancke, L., Siegenthaler, S., Preis, S., \& Steinmetz, H. (2006). Decreased white-matter density in a left-sided frontotemporal network in children with developmental language disorder: Evidence for anatomical anomalies in a motor language network. Brain and Language, 102, 91-98.

Joanisse, M. F., \& Seidenberg, M. S. (1998). Specific language impairment in children: An impairment in grammar or processing? Trends in Cognitive Sciences 2, 240-246.

Joanisse, M. F., \& Seidenberg, M. S. (2003). Phonology and syntax in specific language impairment: Evidence from a connectionist model. Brain and Language, 86, 40-56.

Kemény, F., \& Lukács, A. (2010). Impaired procedural learning in language impairment: Results from probabilistic categorization. Journal of Clinical and Experimental Neuropsychology, 32, 249-258.

Khomsi, A. (2001). Evaluation du Langage Oral. Paris: ECPA

Knowlton, B. J., Mangels, J. A., \& Squire, I. R. (1996). A neostriatal habit learning system in humans. Sciences, 273, 1399-1402.

Lecocq, P. (1998). Epreuve de compréhension syntaxico-sémantique: Adaptation française du TROG: Reception of Grammar Test. Villeneuve d'Ascq, France: Presses Universitaires du Septentrion.

Leonard, L. B. (1998). Children with specific language impairment. Cambridge, MA: MIT Press.

Leonard, L. B., Bortolini, U., Caselli, M. C., McGregor, K. K., \& Sabbadini, L. (1992). Morphological deficits in children with specific language impairment: The status of features in the underlying grammar. Language Acquisition, 2, 151-179.

Leonard, L. B., Ellis Weismer, S., Miller, C. A., Francis, D. J., Tomblin, J. B., \& Kail, R. V. (2007). Speed of processing, working memory, and language impairment in children. Journal of Speech, Language, and Hearing Research, 50, 408-428.

Lum, J. \& Bleses, D. (2012). Declarative and procedural memory in Danish speaking children with specific language impairment. Journal of Communication Disorders, 45, 46-58.

Lum, Conti-Ramsden, Page, \& Ullman (2012). Working, declarative and procedural memory in specific language impairment. Cortex, 48, 1138-1154.

Lum, J., Gelgec, C., \& Conti-Ramsden, G. (2010). Procedural and declarative memory in children with and without specific language impairment. International Journal of Language \& Communication Disorders, 45, 96-107.

Marton, K., Kelmenson, L., \& Pinkhasova, M. (2007). Inhibition control and working memory capacity in children with SLI. Psychologia, 50, 110-121.

McArthur, G. M., \& Bishop, D. (2004). Which people with specific language impairment have auditory processing deficits? Cognitive Neuropsychology, 21, 79-94.

Mengler, E. D., Hogben, J. H., Mitchie, P. T., \& Bishop, D. V. M. (2005). Poor frequency discrimination is related to oral language disorder in children: A psychoacoustic study. Dyslexia, 11, $155-173$.

Meulemans, T., Van der Linden, M., \& Perruchet, P. (1998). Implicit sequence learning in children. Journal of Experimental Child Psychology, 69, 199-221. 
Gabriel et al.: Procedural learning in children with SLI

Miller, C. A., Kail, R., Leonard, L. B., \& Tomblin, J. B. (2001). Speed of processing in children with specific language impairment. Journal of Speech, Language, and Hearing Research, 44, 416-433.

Nissen, M. J., \& Bullemer, P. (1987). Attentional requirements of learning: Evidence from performance measures. Cognitive Psychology, 19, 1-32.

Noterdaeme, M., Amorosa, H., Mildenberger, K., Sitter, S., \& Minow, F. (2001). Evaluation of attention problems in children with autism and children with a specific language disorder. European Child \& Adolescent Psychiatry, 10, 58-66.

Ors, M. (2002). Commentary: Time to drop "specific" in "specific language impairment." Acta Paediatrica, 91, 1025-1030.

Plante, E., Gomez, R., \& Gerken, L. (2002). Sensitivity to word order cues by normal and language/learning disabled adults. Journal of Communication Disorders, 35, 453-462.

Powell, R. P., \& Bishop, D. V. M. (1992). Clumsiness and perceptual problems in children with specific language impairment. Developmental Medicine and Child Neurology, 34, 755-765.

Saffran, J., Johnson, E., Aslin, R., \& Newport, E. (1999). Statistical learning of tone sequences by human infants and adults. Cognition, 70, 27-52.

Schvaneveldt, R. W., \& Gomez, R. L. (1998). Attention and probabilistic sequence learning. Psychological Research/Psychologische Forschung, 61, 175-190.

Schwartz, R. G. (2009). Specific language impairment. In R. G. Schwartz (Ed.), Handbook of child language disorders (pp. 3-43). New York: Psychology Press.

Spaulding, T. J., Plante, E., \& Vance, R. (2008). Sustained selective attention skills of preschool children with specific language impairment: Evidence for separate attentional capacities. Journal of Speech, Language, and Hearing Research, 51, 16-34.

Squire, L. R., Knowlton, B., \& Musen, G. (1993). The structure and organization of memory. Annual Review of Psychology, 44, 453-495.

Tallal, P. (2000). Experimental studies of language learning impairments: From research to remediation. In V. D. M. Bishop \& L. B. Leonard (Eds.), Speech and language impairments in children: Causes, characteristics, intervention and outcome (pp. 131-155). Hove: Psychology Press.

Tallal, P., Merzenich, M., Miller, S. \& Jenking, W. (1998). Language learning impairments: Integrating basic science, technology and remediation, Experimental Brain Research, 123, 210-219.

Tallal, P. \& Piercy, M. (1974). Developmental aphasia: Rate of auditory processing and selective impairment of consonant perception. Neuropsychologia, 12, 83-93.

Tallal, P., \& Stark, R. E. (1981). Speech acoustic-cue discrimination abilities of normally developing and language-impaired children. Journal of the Acoustical Society of America, 69, 568-574.

Tallal, P., Stark, R., \& Mellits, E. D. (1985). Identification of language-impaired children on the basis of rapid perception and production skills. Brain \& Language, 25, 314-322.

Thomas, K., \& Nelson, C. (2001). Serial response time learning in preschool- and school-age children. Journal of Experimental Child Psychology, 79, 364-387.

Tomblin, B., Mainela-Arnold, E., \& Zhang, X. (2007). Procedural learning in children with and without specific language impairment. Journal of Child Language Learning and Development, 3, 269-293.

Ullman, M. T., \& Gopnik, M. (1999). Inflectional morphology in a family with inherited specific language impairment. Applied Psycholinguistics, 20, 51-117.

Ullman, M. T., \& Pierpont, E. (2005). Specific language impairment is not specific to language: The procedural deficit hypothesis. Cortex, 41, 399-433.

Wechsler, D. (2005). Echelle d'intelligence de Wechsler pour enfants et adolescents-Quatrième édition-WISC IV. Paris: ECPA.

Willingham, D. B. (1998). A neuropsychological theory of motor skill learning. Psycholinguistic Review, 105, 558-584. 